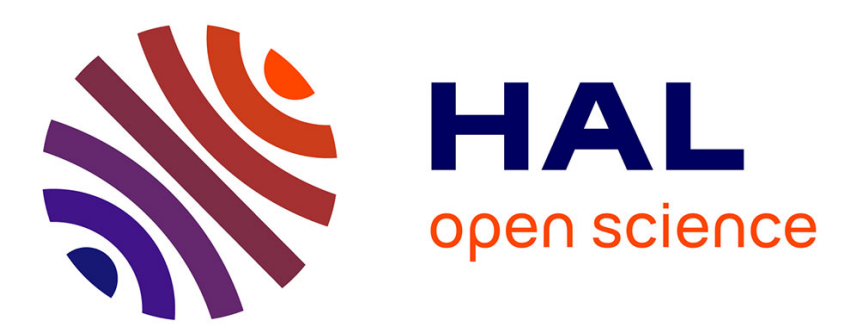

\title{
Structure of the specific combining ability between two species of Eucalyptus. I. RAPD data
}

\author{
C. P. Baril, D. Verhaegen, Ph. Vigneron, J. M. Bouvet, A. Kremer
}

\section{To cite this version:}

C. P. Baril, D. Verhaegen, Ph. Vigneron, J. M. Bouvet, A. Kremer. Structure of the specific combining ability between two species of Eucalyptus. I. RAPD data. TAG Theoretical and Applied Genetics, 1997, 94, pp.796-803. cirad-00845844

\section{HAL Id: cirad-00845844 http://hal.cirad.fr/cirad-00845844}

Submitted on 18 Jul 2013

HAL is a multi-disciplinary open access archive for the deposit and dissemination of scientific research documents, whether they are published or not. The documents may come from teaching and research institutions in France or abroad, or from public or private research centers.
L'archive ouverte pluridisciplinaire HAL, est destinée au dépôt et à la diffusion de documents scientifiques de niveau recherche, publiés ou non, émanant des établissements d'enseignement et de recherche français ou étrangers, des laboratoires publics ou privés. 


\author{
C. P. Baril · D. Verhaegen · Ph. Vigneron \\ J. M. Bouvet • A. Kremer
}

\title{
Structure of the specific combining ability between two species of Eucalyptus. I. RAPD data
}

Received: 3 November 1996 / Accepted: 8 November 1996

\begin{abstract}
Within the context of the reciprocal recurrent selection scheme developed in 1989 by CIRAD-Forêt on Eucalyptus, RAPD essays were performed to assess the genetic diversity in the two species E. urophylla and E. grandis. The molecular markers were split into two parts: the specific markers (present with different frequencies in the two species) and the common markers (present with similar frequencies in the two species). The study analyses the structure of genetic diversity within and between the two species of Eucalyptus. Different genetic distances are worked out for use in prediction equations of the individual tree trunk volume of hybrids at 38 months. Each distance is expressed as the sum of the general genetic distance and the specific genetic distance. The general genetic distance based on the double presence plus the double absence of bands seems to be an interesting co-variate to use in a factor regression model. Through this model the distance calculated between species explains the general combining ability (GCA) and the specific combining ability (SCA) of the phenotypic character with a global coefficient of determination of $81.6 \%$.
\end{abstract}

Key words RAPD - Genetic distances - Heterosis breeding - Prediction - Eucalyptus - Interaction

Communicated by P. M. A. Tigerstedt

C. P. Baril $(\triangle)^{1} \cdot$ D. Verhaegen $\cdot$ Ph. Vigneron - J. M. Bouvet Centre de coopération internationale en recherche agronomique pour le développement, CIRAD-Forêt, BP 5035,

F-34032 Montpellier Cedex 1, France

\footnotetext{
A. Kremer

Institut national de recherche agronomique, INRA, BP 45,

Pierroton, F-33610 Cestas, France

Present address:

${ }^{1}$ Groupe d'étude et de contrôle des variétés et des semences, GEVES, La Minière F-78285 Guyancourt Cedex, France
}

\section{Introduction}

Eucalyptus which originated in Australia, is a highly polymorphic genus (around 600 species) belonging to the family Myrtaceae which is primarily grown for firewood and wood pulp (mainly for paper). It was introduced in the Congo during the fifties and today represents the second major resource of this country. The fortuitous observation of a natural hybrid between two species from the sub-genus Symphomyrtus, namely Eucalyptus urophylla and E. grandis, revealing an important heterosis, led breeders to use a reciprocal recurrent selection scheme (Vigneron 1991). This strategy is especially adapted to interspecific crosses (Gallais 1990) between two highly divergent and complementary populations that have evolved in different environments, with no genetic exchanges between them. E. urophylla, for which 85 samples were collected, is adapted to local conditions and is high-yielding, while E. grandis, for which 25 samples were collected, is less well adapted but has high growth potential.

Since the selection process in tree improvement is very time consuming, any means of predicting tree performance has to be considered with interest. The information required for the effective breeding of hybrids felled at 7 years, can be obtained after 2 or 3 years (Bouvet and Vigneron 1995), but one question remains: which crosses have to be made among all the possible ones? The aim of this paper is to answer this question using genetic distances obtained through the use of RAPDs.

\section{Materials and methods}

In 1990, 13 maternal trees of E. urophylla were crossed with 13 paternal trees of E. grandis in a factorial mating design (Bouvet and Vigneron 1995). While the maternal trees came from two highly differentiated provenances in the island of Flores, namely Monte Lewotobi and Monte Egon, all the pollen came from trees uniformly 
distributed over the northern part of the natural area near Atherton, Queensland, Australia. Unfortunately, technical problems in controlled pollination prevented the mating design from being complete and balanced, with only 87 families among the 169 possible ones (i.e. a ratio equal to $51 \%$ ). Moreover, the number of replicates varied from one to four according to the cross. These constraints led to a reduced factorial design involving nine $E$. urophylla and nine $E$. grandis in which 49 families were present among the 81 possible ones (i.e. a ratio equal to $60 \%$ ) with three or four replications per family. In this reduced design, each female and male parent is represented by at least three or four families, respectively. In each experimental unit (square plot of $4 \times 4=16$ trees), height and circumference at $1.3 \mathrm{~m}$ were measured at 38 months. In the Congo, this corresponds to the half-rotation age in commercial plantations. Volume was calculated by considering the tree trunk as a cone. All of the following analyses were performed using the means of the three or four family replications.

\section{RAPD assays}

The details of total genomic DNA isolation from dry leaves and DNA amplification is presented in Verhaegen et al. (1995). Oligonucleotide primers (10-mers) were used as single primers for the amplification of RAPD sequences according to Williams et al. (1990). The occurrences of a specific band of amplified DNA is scored as 1 and absence as 0 , leading to the characterization of each individual by a binary variable. Pairwise comparisons of individuals were employed to calculate two similarity coefficients: Jaccard's coefficient (Jaccard 1908; Jain et al. 1994) and Sokal and Michener's coefficient (Sokal and Michener 1958), also called a simple matching coefficient (Skroch et al. 1992; Lamboy 1994). From these similarity coefficients, two indexes of dissimilarity between individuals A and B were calculated:

$D_{2}=1-\operatorname{Sim}_{1}$,

where $\operatorname{Sim}_{1}=N_{a b} / N_{a}+N_{b}-N_{a b}, N_{a b}$ is the number of fragments shared by A and B (double presence), while $N_{a}$ and $N_{b}$ are the number of fragments present in individual $\mathrm{A}$ and in individual $\mathrm{B}$, respectively.

$D_{2}=1-\operatorname{Sim}_{2}$,

where $\operatorname{Sim}_{2}=N_{A B} / N, N_{A B}$ is the number of fragments present and absent for individuals $\mathrm{A}$ and $\mathrm{B}$ (double presence plus double absence), and $N$ is the total number of fragments.

Note that $N \times D_{2}$ is the euclidian distance between two individuals.

Let $d^{2}\left(i, i^{\prime}\right)$ be the Euclidian distance between individuals $i$ and $i^{\prime}$, let $j=1, \ldots, N$. Then:

$d^{2}\left(i, i^{\prime}\right)=\sum_{j}\left(x_{i j}-x_{i^{\prime} j}\right)^{2}$,

where $x_{i j}=0$ if the band corresponding to the $j^{\text {th }}$ marker is absent and $x_{i j}=1$ if the band is present. Consequently $x_{i j}-x_{i^{\prime} j}=0$ either if $x_{i j}=x_{i^{\prime} j}=0$ (double absence) or if $x_{i j}=x_{i^{\prime} j}=1$ (double presence). Hence $d^{2}\left(i, i^{\prime}\right)$ is the number of non-coincidences in the two individuals of 0 or 1 .

From the sample of 26 parents (13 E. urophylla and 13 E. grandis), 415 reliable RAPD bands were obtained using 15 primers.

\section{Structure of the genetic diversity}

Firstly, factorial analysis of distance tables seems to be a good tool for visualizing the structure of the genetic diversity. Secondly, in each population each random amplified product can present a number of bands varying from zero (if no individual exhibits the frag-
Table 1 Breakdown table of the number of markers presenting specific combinations of band frequencies in the two Eucalyptus populations (E. urophylla in rows and E. grandis in columns). Italic numbers symbolize the six different groups of markers

$\begin{array}{llllllllllllll}0 & 1 & 2 & 3 & 4 & 5 & 6 & 7 & 8 & 9 & 10 & 11 & 12 & 13\end{array}$

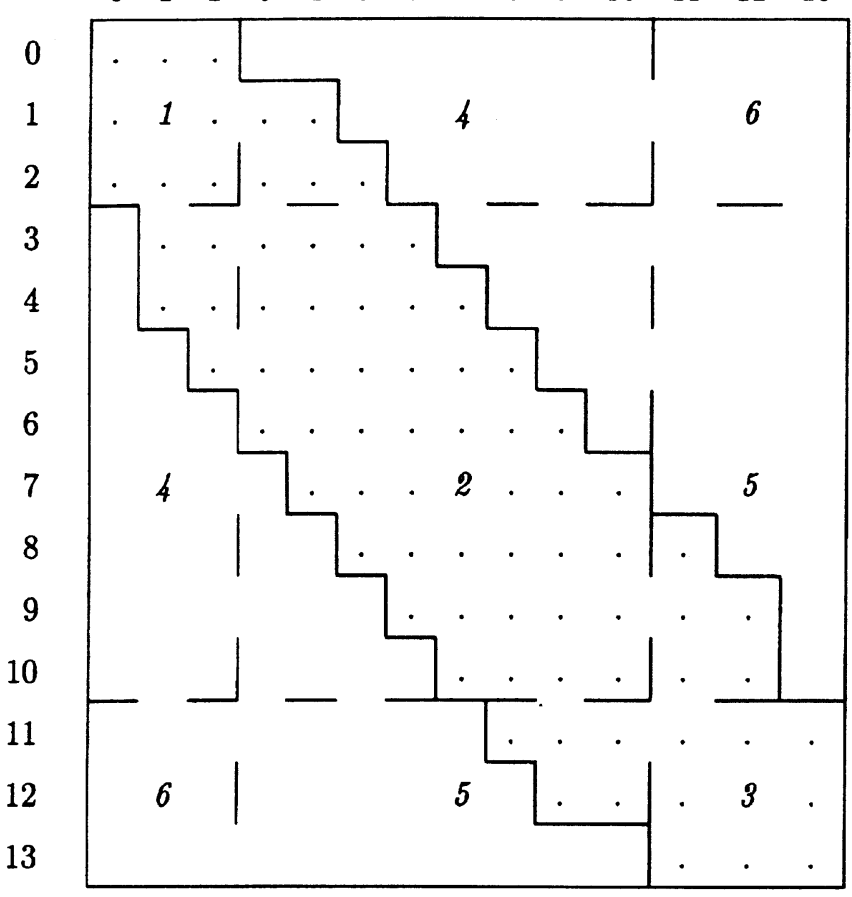

ment) to 13 (if all the individuals show the fragment). A two-way table is constructed with $14 \times 14$ cells corresponding to the coincidence of a specific frequency of bands in the female population with a specific frequency of bands in the male population. The contingency table shows the number of variables concerned with each combination of band frequencies. An exact Fisher test allows one to discriminate markers shared with equal frequency by the two species (dotted area in Table 1) from those showing significant frequency differences between the two species. The contingency table was further subdivided into nine zones corresponding to different values of the exact Fisher test (dashed lines in Table 1). These zones correspond to the combination of three areas in each population, namely the bands whose frequency does not differ significantly from zero [zone (1) if the two species show this type of frequency], the bands whose frequency does not significantly differ from one [zone (3) if the two species show this type of frequency], and the bands with intermediate frequencies [zone (2) if the two species show this type of frequency]. Reciprocal areas have been merged in order to create six different zones (given in italic letters).

Prediction of specific crossing values

The two genetic distances $D_{1}$ and $D_{2}$ have been calculated between and within species using all the RAPD variables, and also using separately either the common variables or the specific variables. Eighteen different distances [six classes of variables $\times$ (two intraspecific distances + one interspecific distance)] have been defined and calculated on the $9 \times 9$ contingency table corresponding to the reduced design. Each distance table has been adjusted to the additive model (1) and the estimates of the additive parameters have been retained as co-variates to be introduced in a factor regression model 
in order to explain GCA (general combining ability) and SCA (specific combining ability). The additive model with two factors applied to distance variables is:

$X_{i j}=\mu+Y_{i}+Z_{j}+R_{i j}$

where $X_{i j}$ is the genetic distance between individual $i$ and individual $j, \mu$ is the general mean distance, $Y_{i}$ is the mean distance of individual $i$ minus the general mean, $Z_{j}$ is the mean distance of individual $j$ minus the general mean and $R_{i j}$ is the residual term.

A large positive or negative $Y_{i}$ indicates that the individual possesses many bands that occur at low or high frequencies, respectively, in the other individuals. If individuals $i$ and $j$ possesses the same bands with low frequencies their $R_{i j}$ will be highly negative, whereas if two individuals possess different bands with low frequencies their $R_{i j}$ will be highly positive. This partition has earlier been proposed on Rogers' distance with RFLP data by Melchinger et al. $(1990 \mathrm{a}, \mathrm{b})$. These general genetic distances can be defined between and within species. In the case of distance tables within species, the null diagonal has been removed before adjustment to the additive model. In this model the interaction term is merged into the residual term. The estimates of additive parameters $Y_{i}$ and $Z_{j}$ are then used as co-variates in the factor regression model (Denis 1988; Baril 1992) applied to the phenotypic variable:

$$
W_{i j}=\mu+\gamma \cdot Y_{i}+\alpha_{i}^{\prime}+\delta \cdot Z_{j}+\beta_{j}^{\prime}+\rho \cdot Y_{i} \cdot Z_{j}
$$$$
+v_{j} \cdot Y_{i}+\lambda_{i} \cdot Z_{j}+\varepsilon_{i j}
$$

where $W_{i j}$ is the individual tree trunk volume at 38 months of the family descending from the cross between mother $i$ and father $j, \mu$ is the general mean, $\alpha_{i}=\gamma \cdot Y_{i}+\alpha_{i}^{\prime}$ is the GCA of mother $i$, $\beta_{j}=\delta \cdot Z_{j}+\beta_{j}^{\prime}$ is the GCA of father $j$, and $\rho \cdot Y_{i} \cdot Z_{j}+v_{j} \cdot Y_{i}+\lambda_{i} \cdot Z_{j}$ is that part of the SCA explained by the model.

Unlike co-variance analysis in which the co-variates depend on the two factors, factor regression allows the partitioning of SCA into functions of only one factor, each multiplied by a regression coefficient depending on the other factor. In order to keep the logical symmetry between the two parents, the co-variates have always been introduced in the model by pairs, i.e. one for E. urophylla and the other for E. grandis. This model provides both an explanation of the additive part of the variability (i.e. GCA) and an explanation of the interactive part (i.e. SCA). Each parental additive contribution to the whole variability is split into two terms. The first represents that part of GCA explained by the regression over the female (or male) co-variate and the second is the rest of GCA (not explained by the general distance). The interaction between the two parents is split into four terms. The first term is the combined regression over the product of the two co-variates (male and female); the two following terms are the remaining regressions over the female co-variate on the one hand and the male co-variate on the other hand. Finally, the rest, $\varepsilon_{i j}$, is the residual term.

The application of models with many parameters when there are numerous missing values in the data set can give fallacious estimates (Denis and Baril 1992). In order to avoid aberrant estimates of phenotypic data, the missing values were estimated by the bi-joint regression model. This model is named by analogy with the joint regression model, well known in studies of genotype $\times$ environment interactions (Finlay and Wilkinson 1963), which allows one to regress the interaction term on one of the two additive terms (the environment effect). In the bi-joint regression model, the interaction between the two factors, namely father and mother, is split into four parts as in a factor regression model where the co-variates depending on each factor are the estimates of the two additive effects (GCA). The first part of the interaction is the regression on the product of the two GCAs, which corresponds to the model proposed by Tukey (1949).

The bi-joint regression model applied to the phenotypic variable gives:

$W_{i j}=\mu+\alpha_{i}+\beta_{j}+\rho \cdot \hat{\alpha}_{i} \cdot \hat{\beta}_{j}+v_{j} \cdot \hat{\alpha}_{i}+\lambda_{i} \cdot \hat{\beta}_{j}+\varepsilon_{i j}$
As for the simple joint regression, the data are first adjusted to the additive model in order to estimate the additive parameters (namely, $\hat{\alpha}_{i}$ and $\widehat{\beta}_{j}$ ), and then adjusted to the complete model using these estimations as co-variates. The bi-joint regression model not only fits the data well (coefficient of determination $=90.8 \%$ ) but also provides estimates which retain part of the interaction. Once the missing values were estimated by this model, the factor regression model has been used on the new completed data set. The estimates of missing data have also been obtained through the additive model, whose results are not presented in this paper, and have given almost similar results in the following analyses. All these analyses were performed using the computer package INTERA (Decoux and Denis 1991), which provides least-squares estimates of parameters. Finally, factorial analyses of the two distance tables $D_{1}$ and $D_{2}$ were performed with a Statistical Analysis System (SAS Institute 1988).

\section{Results}

\section{Structure of the genetic diversity}

Factorial analyses of the two distance tables $D_{1}$ and $D_{2}$ show a clear aggregation of individuals for each species. The first, second and third principal factors, based on $D_{1}$ distance, account for $24.9 \%, 8.6 \%$ and $8.0 \%$, respectively (that is to say a sum equal to $41.5 \%$ ), while the three principal factors, based on $D_{2}$ distance, account for $33.9 \%, 8.2 \%$ and $7.9 \%$, respectively (that is to say a sum equal to $50 \%$ ). The plots of principal factors worked out from the two genetic distances show a tendency for the $D_{2}$ distance (Fig. 1) to provide the

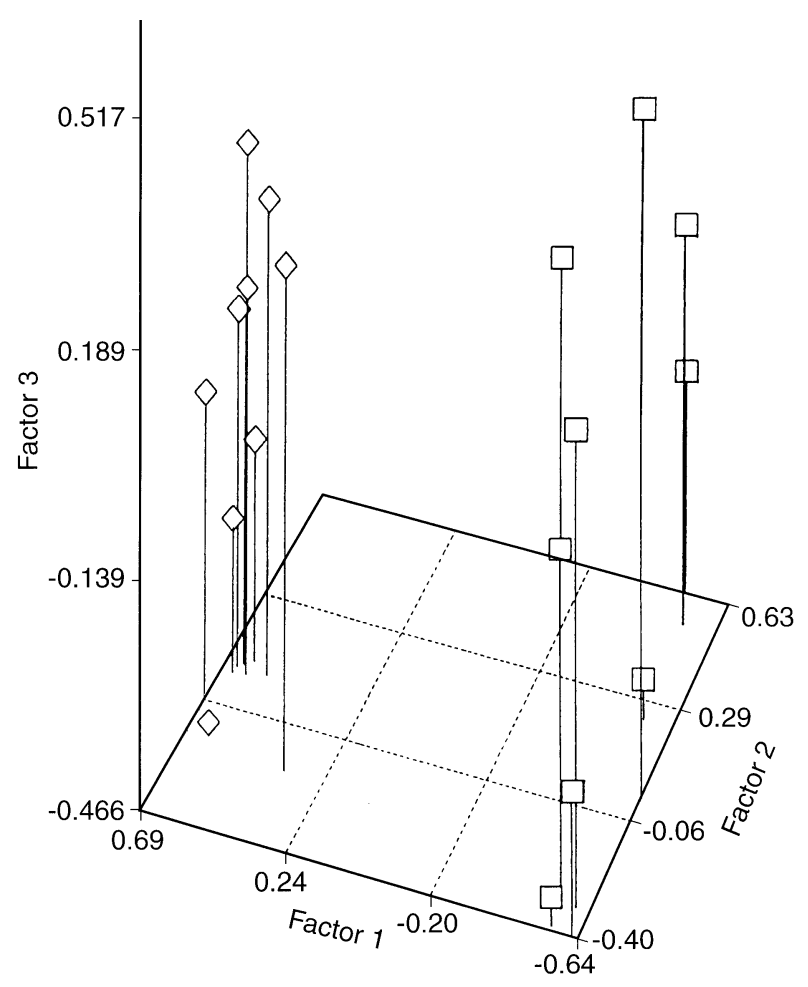

Fig. 1 Plot of the three first factors of FADT computed from $D_{2}$. Species: $\square$ E. urophylla, $\diamond$ E. grandis 
Table 2 Contingency table of the number of markers presenting specific combinations of band frequencies in the two Eucalyptus populations (urophylla in rows and grandis in columns). $\sum_{u}$ and $\sum_{g}$ are the sum of RAPD markers with a particular frequency of bands in E. urophylla and E. grandis, respectively

\begin{tabular}{|c|c|c|c|c|c|c|c|c|c|c|c|c|c|c|c|}
\hline & 0 & 1 & 2 & 3 & 4 & 5 & 6 & 7 & 8 & 9 & 10 & 11 & 12 & 13 & $\sum_{u}$ \\
\hline 0 & - & 15 & 16 & 17 & 6 & 5 & 7 & 5 & 2 & 1 & 1 & 1 & 1 & 2 & 79 \\
\hline 1 & 39 & 13 & 8 & 6 & 4 & 2 & 2 & 0 & 0 & 1 & 2 & 0 & 1 & 0 & 78 \\
\hline 2 & 15 & 8 & 4 & 3 & 3 & 1 & 0 & 2 & 0 & 1 & 1 & 0 & 0 & 0 & 38 \\
\hline 3 & 11 & 10 & 5 & 1 & 1 & 2 & 4 & 0 & 1 & 2 & 1 & 0 & 0 & 1 & 39 \\
\hline 4 & 9 & 4 & 4 & 2 & 2 & 0 & 4 & 0 & 1 & 3 & 1 & 2 & 3 & 0 & 35 \\
\hline 5 & 11 & 4 & 2 & 3 & 2 & 3 & 2 & 0 & 0 & 2 & 0 & 1 & 2 & 1 & 33 \\
\hline 6 & 6 & 5 & 3 & 1 & 0 & 0 & 2 & 0 & 0 & 1 & 0 & 2 & 0 & 1 & 21 \\
\hline 7 & 5 & 1 & 2 & 1 & 1 & 2 & 2 & 0 & 1 & 2 & 0 & 2 & 1 & 0 & 20 \\
\hline 8 & 4 & 1 & 2 & 0 & 2 & 1 & 0 & 1 & 0 & 2 & 2 & 1 & 3 & 0 & 19 \\
\hline 9 & 1 & 1 & 1 & 2 & 1 & 4 & 0 & 0 & 3 & 1 & 0 & 0 & 0 & 0 & 14 \\
\hline 10 & 1 & 1 & 0 & 1 & 0 & 0 & 0 & 0 & 0 & 1 & 1 & 0 & 0 & 0 & 5 \\
\hline 11 & 2 & 1 & 1 & 0 & 0 & 1 & 0 & 1 & 0 & 0 & 1 & 2 & 1 & 4 & 14 \\
\hline 12 & 4 & 0 & 0 & 0 & 1 & 1 & 1 & 0 & 2 & 1 & 1 & 0 & 1 & 1 & 13 \\
\hline 13 & 0 & 0 & 1 & 0 & 0 & 0 & 0 & 0 & 0 & 1 & ? & 1 & 0 & 2 & 7 \\
\hline$g$ & 108 & 64 & 49 & 37 & 23 & 22 & 24 & 9 & 10 & 19 & 13 & 12 & 13 & 12 & 415 \\
\hline
\end{tabular}

best differentiation index between the two species of Eucalyptus. Each cell of the contingency table presented in Table 1 is filled in Table 2 with the number of bands with a specific combination of frequencies in the E. urophylla population and the E. grandis population.

The contingency table is split in two parts, one along the second bisecting line which contains the common markers (dotted area with 226 RAPD variables, i.e. $54.5 \%$ of the total number of markers) and the remaining parts of the table which contain the specific markers (189 RAPD variables, i.e. $45.5 \%$ of the marker sample). Among these markers, 99 are significantly more frequent in the E. urophylla population, and 90 are more frequent in the E. grandis population. The threshold values of the exact Fisher tests were calculated at the $25 \%$ probability level in order to split the markers into two balanced parts, namely the specific ones (with different frequencies for the two species, DIF) and the common ones (with common frequencies for the two species, COM). The sum of markers present in each row ( $\sum_{u}$ for a particular proportion of bands in E. urophylla) and column $\left(\sum_{g}\right.$ for a particular proportion of bands in E. grandis) is shown in the margin of Table 2. For instance, 108 markers were present in at least one $E$. urophylla tree and never in E. grandis trees. The reciprocal situation concerns only 79 markers, never present in E. urophylla. These results are consistent with Fig. 1 which shows a larger variability of the urophylla population when the distance table is based on $D_{2}$, i.e. when the calculation of the distance between two individuals depends on the whole sample of individuals. The lower
Table 3 Frequency of RAPD variables in the nine areas of the contingency table according to the exact Fisher tests calculated at the $P$-level $=25 \%$ (a) and $5 \%$ (b). $p_{u}$ (or $p_{q}$ ) are the band frequencies among E. urophylla (or among E. grandis)

a

\begin{tabular}{llll}
\hline & $p_{g} \simeq 0$ & $0<p_{g}<1$ & $p_{g} \simeq 1$ \\
\hline$p_{u} \simeq 0$ & (1) $28 \%$ & (4) $17 \%$ & (6) $1 \%$ \\
$0<p_{u}<1$ & (4) $23 \%$ & (2) $17 \%$ & (5) $5 \%$ \\
$p_{u} \simeq 1$ & (6) $2 \%$ & (5) $3 \%$ & (3) $3 \%$ \\
\hline $\mathbf{b}$ & & & \\
\hline & $p_{g} \simeq 0$ & $0<p_{g}<1$ & $p_{g} \simeq 1$ \\
\hline$p_{u} \simeq 0$ & (1) $50 \%$ & (4) $9 \%$ & (6) $6 \%$ \\
$0<p_{u}<1$ & (4) $13 \%$ & (2) $3 \%$ & (5) $6 \%$ \\
$p_{u} \simeq 1$ & (6) $5 \%$ & (5) $3 \%$ & (3) $5 \%$ \\
\hline
\end{tabular}

distances between grandis individuals reflect the higher number of coincidences of zero in the 415 RAPD variables relatively to the urophylla individuals. The contingency table was further subdivided in nine parts (dashed lines in Table 1) according to whether or not the frequency of bands is significantly different from 0 or 1 . The proportion of markers present in the each zone is shown in Table 3 (a). The six different groups (in italic letters in Table 1), defined the merging of the symmetric area, from number one to number six: 118 $(28 \%), 72(17 \%), 12(3 \%), 166(40 \%), 33(8 \%)$ and 14 (3\%) RAPD variables.

Common variables with low band frequencies are represented in high frequency (group 1) and comprise those present once either in E. urophylla (39 of them) or in E. grandis (15 of them). In order to prevent the intervention of artefacts (a source of "noise") in the calculation of genetic distances we worked out the different distances excluding these two cells [namely, $(0,1)$ and $(1,0)]$. In fact, the results obtained with these genetic distances were of no interest and hence will not be mentioned further in this paper. Finally, one may note that the cell $(0,0)$ is structurally empty. The contingency table was then subdivided into nine parts according to the exact Fisher test calculated at the 5\% probability level. The proportion of markers present in each new group is shown in Table $3(b)$. The six different groups corresponding to this new structure contain from number one to number six: $206(50 \%), 14(3 \%), 21$ $(5 \%), 94(23 \%), 36(9 \%)$ and $44(11 \%)$ RAPD variables.

\section{Prediction of specific crossing values}

The specific genetic distance $R_{i j}$ (first model) is analogous to the specific Roger's distance proposed by Melchinger et al. (1990). The correlation coefficients between $R_{i j}$ of distances $D_{1}$ and $D_{2}$ and the SCA of tree 
trunk volume at 38 months are not significant ( 0.07 and 0.05 , respectively). These results justify the use of $Y_{i}$ and $Z_{j}$, the general genetic distances, as co-variates in the factor regression model. Two distance tables within species (suffix URO and suffix GRA) and one distance table between species ( for three groups of RAPD variables: the total number of bands (prefix ALL), the subset of common bands (prefix COM) and the subset of different bands (prefix DIF). The sum of the common bands and the different bands forms the total number of RAPD variables. If all

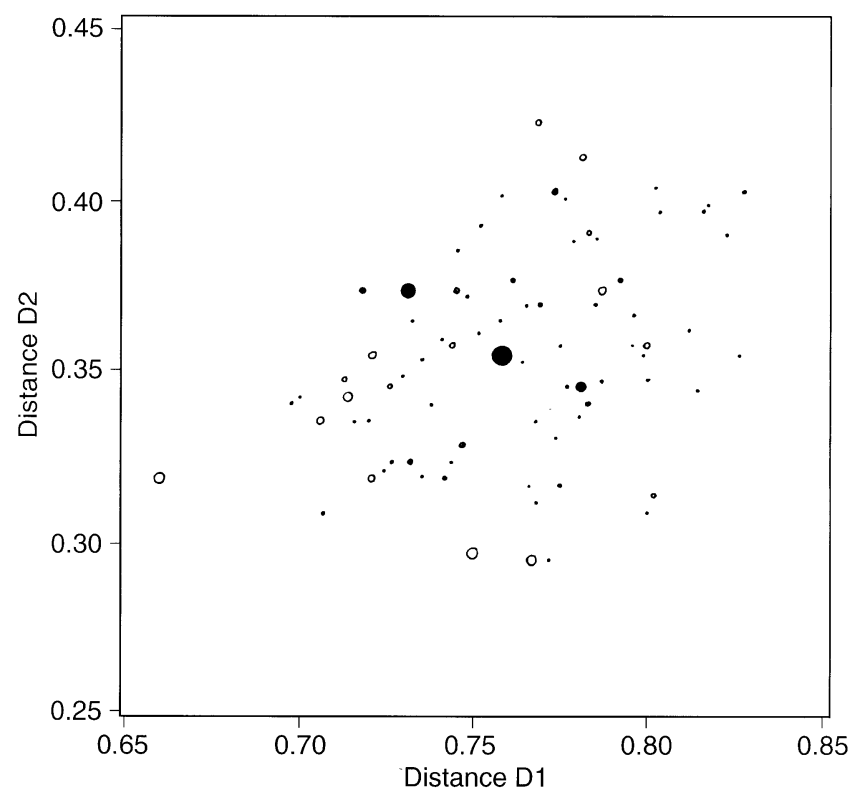

Fig. 2 Plot of genetic distance $D_{2}$ versus $D_{1}$, Circle size stands for the value of SCA; solid circles show a positive SCA and empty bubbles show a negative SCA the bands are considered, the correlation coefficients between distances $D_{1}$ and $D_{2}$ calculated within species (without the null diagonal values) are $\rho=0.77$ and $\rho=0.76$ for $E$. urophylla and E. grandis, respectively. The correlation coefficient between the two types $\left(D_{1}\right.$ and $D_{2}$ ) of between-species distance is 0.36 (significant at the $0.1 \%$ level). This rather small value reflects an actual difference between the two genetic distances. Figure 2 shows the plot of between species $D_{2}$ in terms of between species $D_{1}$ where circle-size stands for he SCA value of tree trunk volume at 38 months. Positive SCAs are solid and negative ones are empty. It seems that highly positive SCA values (heterosis) are confined to medium values of genetic distance.

The additive model was performed on distances $D_{1}$ and $D_{2}$ defined within and between species and worked out from different groups of RAPD variables. The distributions of different sources of variability for each distance are presented in Table 4 with the mean value of each distance table.

The distance tables within species are naturally symmetric and therefore the two additive effects are equal. The amount of variability corresponding to the specific genetic distance $R_{i j}$ is, as expected, greater within species than between species. The greater the amount of the specific genetic distance, the smaller the meaning of the use of general genetic distances. The mean distances within E. urophylla are systematically greater than the mean distances within E. grandis. This remark is especially true for the whole group and a fortiori for the specific group. For these two groups the mean distances between species are clearly greater than the mean distances within species. Finally, the mean distances $D_{2}$, involving double presences plus double absences, are smaller than the mean distances $D_{1}$ which only involve double presences. Correlation coefficients between co-variates associated to the E. urophylla
Table 4 General mean and percentages of the variability of genetic distances explained by the main effects and interaction term. $\% Y_{i}$, $\% Z_{j}$ and $\% R_{i j}$ are the percentages of variability of the distance between individuals $i$ and $j$ explained by the two additive effects and the interaction effect, respectively. When additive effects are not significant (at the 5\% level in the ANOVA) the corresponding percentage of the whole sum of squares is replaced by the NS. $\mu_{D_{1}}$ and $\mu_{D_{2}}$ are the general means of $D_{1}$ and $D_{2}$, respectively, for each distance table

\begin{tabular}{|c|c|c|c|c|c|c|c|c|c|}
\hline \multirow[t]{2}{*}{ Item } & & \multicolumn{4}{|l|}{$D_{1}$} & \multicolumn{4}{|l|}{$D_{2}$} \\
\hline & & $\% Y_{i}$ & $\% Z_{j}$ & $\% R_{i j}$ & $\mu_{D_{1}}$ & $\% Y_{i}$ & $\% Z_{j}$ & $\% R_{i j}$ & $\mu_{D_{2}}$ \\
\hline ALL & $\begin{array}{l}\text { URO } \\
\text { GRA } \\
\mathrm{U} \times \mathrm{G}\end{array}$ & $\begin{array}{l}\text { NS } \\
- \\
44.5\end{array}$ & $\begin{array}{l}- \\
\text { NS } \\
27.3\end{array}$ & $\begin{array}{l}98.3 \\
97.1 \\
28.3\end{array}$ & $\begin{array}{l}0.65 \\
0.61 \\
0.76\end{array}$ & $\begin{array}{l}25.8 \\
- \\
43.4\end{array}$ & $\begin{array}{l}- \\
27.2 \\
35.5\end{array}$ & $\begin{array}{l}48.3 \\
45.6 \\
21.1\end{array}$ & $\begin{array}{l}0.28 \\
0.25 \\
0.35\end{array}$ \\
\hline $\mathrm{COM}$ & $\begin{array}{l}\mathrm{URO} \\
\mathrm{GRA} \\
\mathrm{U} \times \mathrm{G}\end{array}$ & $\begin{array}{l}30.7 \\
- \\
36.9\end{array}$ & $\begin{array}{l}- \\
25.8 \\
14.2\end{array}$ & $\begin{array}{l}38.6 \\
48.9 \\
48.9\end{array}$ & $\begin{array}{l}0.69 \\
0.67 \\
0.68\end{array}$ & $\begin{array}{l}36.2 \\
- \\
48.3\end{array}$ & $\begin{array}{l}- \\
31.8 \\
23.6\end{array}$ & $\begin{array}{l}27.6 \\
36.3 \\
28.4\end{array}$ & $\begin{array}{l}0.25 \\
0.23 \\
0.24\end{array}$ \\
\hline DIF & $\begin{array}{l}\text { URO } \\
\text { GRA } \\
\mathrm{U} \times \mathrm{G}\end{array}$ & $\begin{array}{l}\text { NS } \\
- \\
55.9\end{array}$ & $\begin{array}{l}- \\
27.5 \\
31.5\end{array}$ & $\begin{array}{l}65.4 \\
45 \\
12.6\end{array}$ & $\begin{array}{l}0.61 \\
0.55 \\
0.82\end{array}$ & $\begin{array}{l}\text { NS } \\
- \\
44.2\end{array}$ & $\begin{array}{l}- \\
\mathrm{NS} \\
42.9\end{array}$ & $\begin{array}{l}75.9 \\
65.5 \\
12.9\end{array}$ & $\begin{array}{l}0.31 \\
0.26 \\
0.49\end{array}$ \\
\hline
\end{tabular}


population $\left(Y_{i}\right)$ and the E. grandis population $\left(Z_{j}\right)$ have been calculated (data not shown).

The low correlation coefficients between $D_{1}$ and $D_{2}$, $\mathrm{COM}$ and DIF, and $\mathrm{W}$ and $\mathrm{B}$, justify the calculation of these parameters. The estimates of additive parameters based on different distance tables are used in the factor regression model to explain the tree trunk volume of hybrids at 38 months (Table 5).

The superiority of the explicative power of the $D_{2}$ distance versus the $D_{1}$ distance is clear. Considering the $D_{2}$ distances, those calculated between species are more explicative than those calculated within species. Moreover, distances calculated within E. grandis never explain any part of the variability of the phenotypic character. The co-variates used in the factor regression model are extracted from the between-species $D_{2}$ distances calculated over all the markers (ALL-U $\times \mathrm{G})$. The corresponding ANOVA table is shown in Table 6.

The estimates of the parameters $\gamma, \delta$ and $\rho$ of this model are all positive. Hence the GCA of tree trunk volume at 38 months is positively correlated with the

Table 5 Results of the factor regression model on the tree trunk volume of hybrids at 38 months using the estimates of general genetic distances as co-variates, giving the percentages of variability explained by the different terms of model. When the effects are not significant (at the 5\% level in the ANOVA) the corresponding genetic distance $D_{2}$. If the additive model is applied to the volume at 38 months, the proportion of variability taken into account by the additive part is $60.5 \%$ for the urophylla parent plus $14.1 \%$ for the grandis parent, and the interaction part (SCA) represents $25.4 \%$. In the factor regression model, these three components are split into regressions on the co-variates and residual terms from the regressions. The explicative power of the two co-variates $Y_{i}$ and $Z_{j}$, reflected by the coefficient of determination, is $81.6 \%$.

Finally, the factor regression model based on the $D_{2}$ between-species distance table calculated with the common bands $(\mathrm{COM}-\mathrm{U} \times \mathrm{G})$ presents a determination coefficient equal to $84.2 \%$ while the determination coefficient corresponding to the $\mathrm{D}_{2}$ between-species distance table calculated with the different bands (DIF$\mathrm{U} \times \mathrm{G})$ is $77.8 \%$.

This results shows that the effective RAPD markers for predicting the performance of Ecualyptus hybrids are those presenting common frequencies in the two species.

percentage of the whole sum of squares is replaced by NS. The interaction term consisting of the regression on the product of the male and female co-variates $\left(\rho \cdot Y_{i} \cdot Z_{j}\right)$ is never significant and hence has not been presented in this table

\begin{tabular}{|c|c|c|c|c|c|c|c|c|c|}
\hline & & \multicolumn{4}{|l|}{$D_{1}$} & \multicolumn{4}{|l|}{$D_{2}$} \\
\hline & & $\% \gamma \cdot Y_{i}$ & $\% \delta \cdot Z_{j}$ & $\% v_{j} \cdot Y_{i}$ & $\lambda_{i} \cdot Z_{j}$ & $\% \gamma \cdot Y_{i}$ & $\% \delta \cdot Z_{j}$ & $\% v_{j} \cdot Y_{i}$ & $\lambda_{i} \cdot Z_{j}$ \\
\hline \multirow{3}{*}{ ALL } & URO & NS & - & NS & - & 18.8 & - & 6.6 & - \\
\hline & GRA & - & NS & - & NS & - & NS & - & NS \\
\hline & $\mathrm{U} \times \mathrm{G}$ & NS & NS & NS & NS & 37.2 & NS & 6.8 & NS \\
\hline \multirow[t]{3}{*}{$\mathrm{COM}$} & URO & NS & - & NS & - & 15.2 & - & NS & - \\
\hline & GRA & - & NS & - & NS & - & NS & - & NS \\
\hline & $\mathrm{U} \times \mathrm{G}$ & 6.1 & 3.5 & NS & NS & 26.9 & 1.8 & 8.2 & NS \\
\hline \multirow[t]{3}{*}{ DIF } & URO & NS & - & NS & - & 18.2 & - & NS & - \\
\hline & GRA & - & NS & - & NS & - & NS & - & NS \\
\hline & $\mathrm{U} \times \mathrm{G}$ & NS & NS & NS & NS & 13.7 & NS & NS & NS \\
\hline
\end{tabular}

Table 6 ANOVA of the factor regression model with one covariate associated to each parent (derived from the ALL$\mathrm{U} \times \mathrm{G} D_{2}$ distance). The last column of the ANOVA table shows the amount of the variability of the phenotypic character explained by each term of the factor regression model

\begin{tabular}{lrrrrrr}
\hline $\begin{array}{l}\text { Source of } \\
\text { variation }\end{array}$ & $\begin{array}{l}\text { Sum of } \\
\text { squares }\end{array}$ & $d f$ & $\begin{array}{c}\text { Mean } \\
\text { squares }\end{array}$ & $F$-test & $P$-level & $\begin{array}{l}\text { \% Sum of } \\
\text { squares }\end{array}$ \\
\hline $\begin{array}{l}\text { E. urophylla } \\
\gamma \cdot Y_{i}\end{array}$ & $\mathbf{4 5 9 7 . 8 2}$ & $\mathbf{8}$ & $\mathbf{5 7 4 . 7 3}$ & - & - & $\mathbf{6 0 . 5 \%}$ \\
$\alpha_{i}^{\prime}$ & 2827.41 & 1 & 2827.41 & 99.1 & $<0.001$ & $37.2 \%$ \\
E. grandis & 1770.41 & 7 & 252.91 & 8.9 & $<0.001$ & $23.3 \%$ \\
$\delta . Z_{j}$ & $\mathbf{1 0 7 2 . 0 3}$ & $\mathbf{8}$ & $\mathbf{1 3 4 . 0 0}$ & - & - & $\mathbf{1 4 . 1 \%}$ \\
$\beta_{j}^{\prime}$ & 1.03 & 1 & 1.03 & 0.0 & 0.844 & $\simeq 0.0 \%$ \\
E. uro $\times$ E. gra & 1070.99 & 7 & 153.00 & 5.4 & $<0.001$ & $14.1 \%$ \\
$\rho \cdot Y_{i} \cdot Z_{j}$ & $\mathbf{1 9 3 4 . 2 6}$ & $\mathbf{8 1}$ & $\mathbf{2 3 . 8 8}$ & - & - & $\mathbf{2 5 . 4 \%}$ \\
$v_{j} \cdot Y_{i}$ & 0.68 & 1 & 0.68 & 0.0 & 0.873 & $\simeq 0.0 \%$ \\
$\lambda_{i} \cdot Z_{j}$ & 517.35 & 7 & 73.91 & 2.6 & 0.023 & $6.8 \%$ \\
$\varepsilon_{i j}$ & 17.56 & 7 & 2.51 & 0.1 & 0.998 & $0.2 \%$ \\
\hline
\end{tabular}




\section{Discussion}

Without any available references concerning the use of RAPDs is Eucalyptus breeding, our approach is mainly prospective.

Firstly, this study shows that genetic distance based on RAPDs provides a useful tool to differentiate the two species of Eucalyptus. If the RAPD variables are clustered in nine different groups according to their frequency in both species tested at the $5 \%$ probability level, bands with low frequencies in both species (group 1) represented $50 \%$ of the total number. This structure of the genetic diversity provides results characterized by a large number of bands with low frequencies and a small number of bands with high frequencies. Surprisingly, the bands predicting hybrid performance are those with similar frequencies common to the two species.

Secondly, the factor regression model gives an interesting partitioning of the GCA and the SCA on tree trunk volume data at 38 months into linear functions of relevant male and female co-variates. The global coefficient of determination, $81.6 \%$, is satisfactory, knowing that the general genetic distance of E. urophylla individuals explains $27 \%$ of SCA of tree trunk volume at 38 months. The prediction of heterosis through genetic markers is an old dream of plant breeders. The usefulness of RAPD-based genetic distance measures in predicting the performance of between-species hybrids in Eucalyptus is thus demonstrated.

Thirdly, this study examines the problem of the choice of genetic distance for predicting crossing values. The best co-variates are defined from genetic distances calculated between the species of Eucalyptus and based on the simple matching coefficient. The greater efficiency of co-variates derived from betweenspecies distance versus co-variates derived from withinspecies distance is intuitively obvious. However, the greater efficiency of co-variates corresponding to general genetic distances defined by the double presence plus the double absence $\left(D_{2}\right)$ of bands is not evident. The two genetic distances $D_{1}$ and $D_{2}$ have been defined at the two extremities of the spectrum of the possible distances with no a priori preference. There are convincing arguments in favour of each definition of genetic distance. Jaccard's coefficient does not depend on the individual samples studied but its use implicitly presupposes a positive effect of the presence of bands. Peltier et al. (1994) compared these two genetic distances using RAPD markers in the polygenetic reconstruction of seven species of Petunia. The authors showed that the best fit between the a priori taxonomy and the a posteriori grouping is obtained with $D_{1}$ for a priori distant species, while the best fit is obtained with $D_{2}$ for a priori near species. Two phenomena can generate the absence of a RAPD band: a modification of a flanking sequence and the entire absence of the amplified se- quence. In the first case, $D_{2}$ is the more accurate genetic distance, while in the second case $D_{1}$ is more accurate. One could argue that it would be interesting to test other distances. Let us consider the weighted Euclidian distance $d^{\prime 2}\left(i, i^{\prime}\right)$ between the two individuals $i$ and $i^{\prime}$ : $d^{\prime 2}\left(i, i^{\prime}\right)=\sum_{j} w_{j}\left(x_{i j}-x_{i^{\prime} j}\right)^{2}$ where $w_{j}=1 / \operatorname{var}\left(V_{j}\right)$ with $\operatorname{var}\left(V_{j}\right)$ the variance of the $j^{\text {th }}$ marker. This distance would emphasize the loci presenting a great imbalance between a proportion of 0 (absence of the band) and a proportion of 1 (presence of the band). The inverse weighting would emphasize the loci with a similar proportion of presence and absence of bands and might be more interesting. Other kinds of weights could be imagined employing different criteria. For example, the number of bases constituting the primers used in the polymerase chain reaction (PCR) could be one of them.

To conclude, this study shows the necessity of using a genetic distance based on common markers for two species in order to predict the value of crosses. Instead of using a large number of RAPD bands, it would be more efficient to find specific bands for the genomic regions actually contributing to heterosis for the agronomic traits of interest. A thorough reflection of the definition of genetic distances and localization in the between-species contingency table of bands linked to quantitative traits will be the subject of a further paper.

\section{References}

Baril CP (1992) Factorial regression for interpreting genotypeenvironment interaction in bread wheat trials. Theor Appl Genet $83: 1022-1026$.

Bouvet JM, Vigneron $\mathrm{Ph}$ (1995) Age trends in variances and heritablities in Eucalyptus matting designs. Silvae Genet $44: 206-216$

Denis JB (1988) Two-way analysis using covariates. Statistics 19: $123-132$

Denis JB, Baril CP (1992) Sophisticated models with numerous missing values: the multiplicative interaction model as an example. Biuletyn Oceny Odmian, Poland 24-25:33-45

Decoux G, Denis JB (1991) INTERA. Logiciels pour l'interprétation statistique de l'interaction entre deux facteurs. Biométrie INRA route de Saint-Cyr F 78026 Versailles France

Finlay KW, Wilkinson GN (1963) The analysis of adaptation in a plant-breeding programme. Aust J Agric Res 14:742-754

Gallais A (1990) Théorie de la sélection en amélioration des plantes. Coll Sci Agro Ed Masson

Jaccard P (1908) Nouvelles recherches sur la distribution florale. Bull Soc Vaud Sci Nat 44:223-270

Jain A, Bhatia S, Banga SS, Prakash S (1994) Potential use of random amplified polymorphic DNA (RAPD) technique to study the genetic diversity in Indian mustard (Brassica juncea) and its relationship to heterosis. Theor Appl Genet 88:116-122

Lamboy WF (1994) Computing genetic similarity coefficients from RAPD data: the effects of PCR artifacts. Cold Spring Harbor Laboratory Press ISSN, 31-37

Melchinger AE, Lee M, Lamkey KR, Woodman WL (1990 a) Genetic diversity for restriction fragment length polymorphisms: relation to estimated genetic effects in maize inbreds. Crop Sci $30: 1033-1040$ 
Melchinger AE, Lee M, Lamkey KR, Hallauer AR, Woodman WL (1990 b) Genetic diversity for restriction fragment length polymorphisms and heterosis for two diallel sets of maize inbreds. Theor Appl Genet 80:488-496

Peltier D, Chacon H, Tersac M, Caraux G, Dulieu H, Berville A (1994) Utilisation des RAPD pour la construction de phylogrammes chez Petunia. In: Techniques et utilisations des marqueurs moléculaires. Coll Les colloques INRA

SAS Instiutute (1988) SAS language guide for personal computers. Release 6.03 edition. SAS Institute, Cary, North Carolina

Skroch P, Tivang J, Nienhuis J (1992) Analysis of genetic relationships using RAPD marker data. Joint plant breeding symposia series: applications of RAPD technology to plant breeding, pp 26-30
Sokal RR, Michener CD (1958) A statistical method for evaluating systematic relationships. Univ Kansas Sci Bull 38:1409-1438

Tukey JH (1949) One degree of freedom for non-additivity. Biometrics $5: 232-242$

Verhaegen D, Kremer A, Vigneron Ph (1995) Relationships between heterosis and molecular polymorphism in interspecific crosses of Eucalyptus urophylla $\times$ Eucalyptus grandis. CRC for temperate hardwood forestry, IUFRO, Hobart, Tasmania, Australia

Vigneron $\mathrm{Ph}$ (1991) Création et amélioration des variétés d'hybrides d'Eucalyptus, Durban, September 1991, pp 345-360

Williams JGK, Kubelik AR, Livak KJ, Rafalski JA, Tingey SV (1990) DNA polymorphisms amplified by arbitrary primers are useful as genetic markers. Nucleic Acids Res 18:6531-6535 